\title{
Does Acute Supplementation with Nitrate-Rich Beetroot Juice Benefit Older Adults More than Younger Adults ${ }^{\dagger}$
}

\author{
Luke Stanaway ${ }^{1}$, Kay Rutherfurd-Markwick ${ }^{2,3}$, Rachel Page 4,5, Marie Wong 3,5, Wannita Jirangrat ${ }^{6}$, \\ Koon Hoong Teh ${ }^{5}$ and Ajmol Ali 1,5,* \\ 1 School of Sport, Exercise and Nutrition, Massey University, Auckland 0755, New Zealand; \\ 1.stanaway@massey.ac.nz \\ 2 School of Health Sciences, Massey University, Auckland 0745, New Zealand; k.j.Rutherfurd@massey.ac.nz \\ 3 Centre for Metabolic Health Research, Massey University, Auckland 0745, New Zealand; \\ m.wong@massey.ac.nz \\ 4 School of Health Sciences, Massey University, Wellington 6140, New Zealand; r.a.page@massey.ac.nz \\ 5 Massey Institute of Food Science and Technology, Massey University, Auckland 0745, New Zealand; \\ kenteh29@yahoo.co.nz \\ 6 Thai Union Group PCL, Bangkok 74000, Thailand; Wannita.Jirangrat@thaiunion.com \\ * Correspondence: a.ali@massey.ac.nz; Tel: 0064-9-213-6414 \\ + Presented at the 2018 Nutrition Society of New Zealand Annual Conference, Auckland, New Zealand, \\ 28-30 November 2018.
}

Published: 11 March 2019

Background: To investigate age-related effects of acute dietary nitrate $\left(\mathrm{NO}_{3}{ }^{-}\right)$supplementation on cardiovascular responses, cognition and mood.

Methods: 13 younger (18-30 y) and 11 older (50-70 y) adults consumed $150 \mathrm{~mL}$ of either $\mathrm{NO}^{-}$ rich beetroot juice (BR; $10.5 \mathrm{mmol} \mathrm{NO}_{3}^{-}$) or placebo solution (PL; $1 \mathrm{mmol} \mathrm{NO}_{3}^{-}$) in a double blind, crossover design, $2.25 \mathrm{~h}$ prior to a 30 -min treadmill walk. Nitrate and nitrite blood concentrations, blood pressure (BP), heart rate (HR), oxygen uptake kinetics, cognitive function, mood and perceptual tests were performed throughout each trial.

Results: BR consumption significantly increased plasma nitrate $(p<0.001)$ and nitrite $(p=0.003)$ concentrations and reduced systolic $(p<0.001)$ and diastolic BP $(p=0.013)$ compared to placebo within each age group. Older adults showed a greater elevation in plasma nitrite $(p=0.038)$ and a greater reduction in diastolic BP $(p=0.005)$ following BR consumption than younger adults. Reaction time was improved in the Stroop test following BR supplementation for both groups $(p=0.045)$. However, there were no main effects of treatment or interaction of treatment, time and age for HR, oxygen uptake, other cognitive tests, and mood or perceptual measures examined following BR supplementation in either age group $(p>0.05)$.

Conclusions: For the first time it has been shown that acute BR supplementation increased plasma nitrite concentration and reduced diastolic BP to a greater degree in older adults; whilst, systolic BP was reduced in both older and younger adults. These results suggest nitrate enriched BR may prove a successful dietary intervention strategy for reducing or delaying development of prehypertension to hypertension.

Supplementary Materials: The PDF of oral presentation is available online at www.mdpi.com/2504-3900/8/1/26/s1.

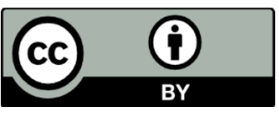

(C) 2019 by the authors. Licensee MDPI, Basel, Switzerland. This article is an open access article distributed under the terms and conditions of the Creative Commons Attribution (CC BY) license (http://creativecommons.org/licenses/by/4.0/). 